\title{
Metabolic evidence for episodic memory plasticity in the nonepileptic temporal lobe of patients with mesial temporal epilepsy
}

\author{
$* \dagger$ Nicola Trotta, $* \dagger$ Serge Goldman, $\ddagger$ Benjamin Legros, *Noémie Ligot, $\ddagger$ Nathalie Guerry, \\ $\S$ Kristof Baete, §Koen Van Laere, *\$Patrick Van Bogaert, and *Xavier De Tiège
}

\begin{abstract}
*Laboratoire de Cartographie Fonctionnelle du Cerveau, Université Libre de Bruxelles, Brussels, Belgium; †Department of Nuclear Medicine, Université Libre de Bruxelles, Brussels, Belgium; \$Reference Centre for Refractory Epilepsy, Hôpital Erasme, Université Libre de Bruxelles, Brussels, Belgium; and §Department of Nuclear Medicine, Universitair Ziekenhuis Leuven, Leuven, Belgium
\end{abstract}

\section{SUMMARY}

Purpose: Metabolic changes have been described in the nonepileptic temporal lobe of patients with unilateral mesiotemporal lobe epilepsy (MTLE) associated with hippocampal sclerosis (HS). To better understand the functional correlate of this metabolic finding, we have sought to characterize brain regions in patients with MTLE that show correlation between unilateral episodic memory performances, as assessed by intracarotid amobarbital test (IAT), and interictal regional cerebral metabolism measured by $\left[{ }^{18} \mathrm{~F}\right]$-fluorodeoxyglucose positron emission tomography (FDG-PET).

Methods: Resting FDG-PET was performed interictally in 26 patients with unilateral MTLE caused by HS ( 16 female, mean age: 36 years; 16 left HS). Using statistical parametric mapping (SPM8), we performed a group comparison analysis comparing brain metabolism in the patients and in 54 adult controls (27 female, mean age: 32 years), with FDG-PET data of right HS patients being flipped. IAT scores of nonepileptic hemisphere functions (amobarbital injection ipsilateral to HS) were used as covariates of interest in a correlation analysis with regional brain metabolism.

Key Findings: The group comparison analysis revealed significant hypometabolic areas in a widespread temporofrontal network ipsilateral to HS. In addition, a significant increase in metabolism was found in mesial and lateral temporal regions contralateral to HS. Significant positive correlations were found between IAT scores of nonepileptic hemisphere functions and mesial temporal metabolism in this hemisphere.

Significance: This study demonstrates the existence of significant increase in relative regional cerebral glucose metabolism in mesial and lateral temporal regions contralateral to the epileptic focus in patients with unilateral MTLE associated with HS. The positive correlation in these brain regions between IAT scores and metabolism supports the role of disease-induced plasticity mechanisms contralateral to HS in the preservation of episodic memory processes.

KEY WORDS: Mesial temporal lobe epilepsy, $\left[{ }^{18} \mathrm{~F}\right]-$ Fluorodeoxyglucose positron emission tomography, Intracarotid amobarbital test, Plasticity.
Mesial temporal lobe epilepsy (MTLE) is a chronic neurologic condition characterized by recurrent partial seizures arising from the mesiotemporal structures; it is associated with hippocampal sclerosis (HS) in 60-80\% of patients (Berkovic et al., 1995; Van Paesschen et al., 1995). MTLE is the most common form of pharmacoresistant partial epilepsy in adults (Rosenow \& Luders, 2001; Schuele \& Luders, 2008). For patients with MTLE whose seizures remain uncontrolled after well-

Accepted August 4, 2011; Early View publication September 20, 2011.

Address correspondence to Ir Nicola Trotta, Laboratoire de Cartographie Fonctionnelle du Cerveau, Hôpital Erasme, Université Libre de Bruxelles, Route de Lennik 808, 1070 Brussels, Belgium. E-mail: ntrotta@ulb.ac.be

Wiley Periodicals, Inc.

(C) 2011 International League Against Epilepsy ordered attempts of antiepileptic drug (AED) regimens, amygdalohippocampectomy associated or not with anterior temporal lobectomy is a widely accepted and effective therapeutic option (Wiebe et al., 2001; Engel et al., 2003). Memory deficits, however, represent a potential complication of temporal lobectomy (Ojemann \& Dodrill, 1985; Elger et al., 2004), due to the key role of temporal structures in memory neuronal networks. Substantial verbal memory decline is observed in about $50-85 \%$ of patients who undergo a resection of the left temporal region (Gleissner et al., 2002, 2004; Helmstaedter et al., 2003; Bonelli et al., 2010). In addition, nonverbal memory deficits can be found in about $30-50 \%$ of patients who undergo right temporal resection (Gleissner et al., 2002, 2004; Helmstaedter et al., 2003; Bonelli et al., 2010). 
The intracarotid amobarbital test (IAT), also known as the Wada test, was integrated 50 years ago into the presurgical evaluation of patients with MTLE who are potential candidates for amygdalohippocampectomy (Wada \& Rasmussen, 1960). This procedure is usually performed to determine the brain hemisphere that is dominant for major cognitive functions such as speech or memory (Spencer et al., 2000). The IAT typically involves the injection of sodium-amobarbital into a single internal carotid artery via a transfemoral arterial catheterization. This procedure suppresses neuronal activity in the anterior and middle cerebral artery territories of the injected hemisphere while the patient is presented with multiple cognitive tasks. The procedure actually challenges the spared vascular territories, in particular in the noninjected hemisphere, for specified cognitive functions (Loring et al., 1990). Impaired memory performance is observed in about $20-30 \%$ of cases after injection into the epileptic hemisphere, whereas this proportion reaches $60-80 \%$ after contralateral injection (Powell et al., 1987; Rausch et al., 1989; Wyllie et al., 1991). Based on these data, several authors advocate IAT performance as a way to predict task-specific memory decline after surgery (and eventually to propose surgical abstention if global amnesia is predicted), and as an adjunct to determine seizure focus laterality (Wyllie et al., 1991; Perrine et al., 1993; Rouleau et al., 1997).

Positron emission tomography studies using $\left[{ }^{18} \mathrm{~F}\right]$-fluorodeoxyglucose (FDG-PET) performed in patients with unilateral MTLE have demonstrated significant hypometabolism in the epileptic temporal lobe of about $60-95 \%$ of the patients (Engel et al., 1990; Henry et al., 1990, 1993; Theodore et al., 1992; Duncan, 1997; Wieser, 2004). Several studies have shown that the existence of focal hypometabolism within the epileptic temporal lobe is associated with a substantial impairment in episodic memory as assessed by IAT with contralateral injection (Salanova et al., 1992; Jokeit et al., 1999). In addition, a significant positive correlation between asymmetry indexes of glucose metabolism in mesial temporal structures and IAT scores has been shown, suggesting that the asymmetry index of glucose metabolism in the hippocampus reflects the degree of memory dominance as revealed by IAT (Hong et al., 2000). Interestingly, some FDG-PET studies performed interictally in patients with unilateral MTLE have shown, in addition to the expected hypometabolism in the epileptic temporal lobe, the existence of substantial increase in metabolism in the mesial and lateral temporal regions contralateral to the seizure focus (Rubin et al., 1995; Van Bogaert et al., 2000). This hypermetabolism contralateral to the seizure focus is interpreted as a reflection of plasticity mechanisms within the nonepileptic temporal lobe, compensating for the disease-related dysfunction in the epileptic temporal lobe.

The purpose of this study is to better understand the functional correlate of the metabolic changes observed in the nonepileptic temporal lobe by characterizing the brain regions showing positive correlation between unilateral episodic memory performances, as assessed by IAT, and interictal cerebral metabolism measured by FDG-PET in a large group of patients with unilateral MTLE associated to HS.

\section{Patients And Methods}

\section{Patients and control subjects}

Among the population of patients studied by FDG-PET between January 2001 and May 2009 at the PET/Biomedical Cyclotron Unit of the ULB-Hôpital Erasme, Belgium, we retrospectively selected 26 patients (16 women, aged 9-58 years, mean age 36.6 years) based on the following inclusion criteria: (1) refractory epilepsy included in the multidisciplinary presurgical evaluation program of the ULB-Hôpital Erasme, (2) 5 days of scalp video-electroencephalography (EEG) suggesting unilateral MTLE, (3) unilateral HS on structural cerebral magnetic resonance imaging (MRI), (4) bilateral IAT performed in the context of the presurgical evaluation, and (5) absence of neurologic condition other than epilepsy. All patients underwent neuropsychological evaluations and FDG-PET. Because patients were retrospectively selected and underwent their presurgical evaluation over an extended period (January 2001-May 2009), the cognitive tests used for the neuropsychological evaluation were not standardized across patients. Sixteen patients had a left MTLE and 10 had a right MTLE. Based on IAT, 21 of the 26 patients were left dominant for speech function, 3 were right dominant, and 2 had bilateral representation. The duration of their epilepsy ranged from 5-54 years, with a mean duration of 26.5 years. Surgical procedures were performed in 23 patients (anterior temporal and amygdalohippocampal disconnection in 19 and radiosurgery in 4). Outcome for seizures (follow-up ranging from 4 months up to 9 years) showed 16 patients (70\%) in Engel class IA, three in Engel class II, and four in Engel class IIIA.

A group of 54 healthy adult volunteers ( 27 women and 27 men aged $17-50$ years, mean age 32.4 years) was used as control population. FDG-PET data of 29 subjects were acquired at the PET/Biomedical Cyclotron Unit of the ULB-Hôpital Erasme. FDG-PET data of the 25 other subjects were acquired at the Nuclear Medicine division of the UZ Leuven. All FDG-PET data were acquired on the same type of PET camera using the same data acquisition procedure. The institutional ethics committees gave approval for these FDG-PET investigations. Written informed consent was obtained from all control subjects.

The patient and control populations included in this study are completely different from those included in Van Bogaert et al. (2000).

The ULB-Hôpital Erasme Ethics Committee gave approval for conducting this retrospective study. 


\section{Intracarotid amobarbital test protocol}

IATs were performed at the Angiography Department of ULB-Hôpital Erasme using a constant methodology adapted from the method of Blume et al. (1973). About 2-3 min before sodium-amobarbital injection, two items (a number and a color) were visually presented during a few seconds, and patients were asked to name them after presentation to make sure that they understood the procedure. Ten seconds before the injection, the patients were asked to count aloud and to raise both arms at the same time. Next, a 4 -s bolus of $2 \mathrm{mg} / \mathrm{kg}$ (maximum $144 \mathrm{mg}$ ) of sodium-amobarbital was injected into the internal carotid artery ipsilateral to the epileptogenic zone, inducing hemiplegia contralateral to the site of injection. Then, 11 consecutive items were presented at intervals of about $30 \mathrm{~s}$ (three visually presented words, two pictures, three actions, two spoken words, and one fantasy picture) and the patients were asked to name every one of them. Language dominance was assessed on the basis of clinical signs of aphasia and inability to name the presented items. After the item presentation, the patient was left in the angiography room for $12 \mathrm{~min}$ to recover from the induced hemiplegia without any extraneous input. Then, memory performances were tested by asking the patient to name all previously presented items spontaneously. If the patient was not able to do it spontaneously, he or she was asked to identify the presented items on six-choice panels. For each patient, IAT score $(0-11)$ was calculated by subtracting false from correct recognitions. The whole procedure was repeated $10-15 \mathrm{~min}$ after the previous one with a carotid artery injection contralateral to the epileptogenic zone. Throughout this article, IAT scores are noted "right" and "left" in reference to the injected side, keeping in mind that the test actually challenges the performance of the contralateral hemisphere. For every patient, IAT scores were normalized by dividing unilateral scores by the sum of the scores obtained from the right and left injections, using the following formula: (unilateral IAT score $)_{\text {norm }}=($ unilateral IAT score $) /($ right IAT score + left IAT score). This normalization procedure aims to adjust the unilateral scores to the global patient performance, reducing the individual variability unrelated to unilateral performance (Akanuma et al., 2009).

Significant differences between left and right IAT scores in the groups of patients with left and right MTLE were calculated using the two-tailed paired $t$-test function of the Matlab statistical toolbox version 6.2 (Matlab 7.6 R2008a; MathWorks Inc., Natick, MA, U.S.A.). Results of these analyses were considered significant at a value of $p<0.05$.

\section{FDG-PET data acquisition}

FDG-PET data were acquired using an ECAT 962 Exact HR+ camera (CTI-Siemens, Knoxville, TX, U.S.A.), the characteristics of which have been described previously (Brix et al., 1997). The patients' antiepileptic drugs (AEDs) were unchanged. All patients fasted for at least $4 \mathrm{~h}$, were awake in an eyes-closed rest, and received an intravenous bolus injection of 2-5 mCi (74-185 Mbq) of FDG before a 20-min PET data acquisition in three-dimensional mode. One emission frame composed of 63 transaxial slices was obtained and realigned to the canthomeatal line. Images were corrected for photon attenuation thanks to a postinjection 10-min transmission scan obtained with Ge-68 line sources. Each PET image was reconstructed using filtered back projection and displayed in a $128 \times 128 \times 63$ voxel format, with a slice thickness of $2.4 \mathrm{~mm}$ and an in-plane resolution of $4.6 \mathrm{~mm}$.

\section{PET data analyses}

FDG-PET data were analyzed using the voxel-based Statistical Parametric Mapping method (SPM8; http://www. fil.ion.ucl.ac.uk/spm/, Wellcome Department of Imaging Neuroscience, London, United Kingdom). The PET images were spatially normalized into the Montreal Neurologic Institute template (Montreal Neurologic Institute, Montreal, QC, Canada). The scans were then smoothed using a 12-mm full-width at half-maximum isotropic kernel. Global activity normalization was performed by proportional scaling (Van Bogaert et al., 2000).

In a first step, subtractive SPM analyses were conducted at the individual level to compare the PET data of each patient with those of the control group (Van Bogaert et al., 2000; De Tiège et al., 2004, 2008). For these analyses, the design matrix built for each patient included the patient's scan and the scans of the 54 control subjects. Separate $t$-contrast analyses identified brain regions where glucose metabolism was significantly lower or higher in each patient than in the control population.

In a second step, subtractive statistical parametric mapping (SPM) analyses were conducted at the group level to compare the PET data of the patients taken as a group with those of the control group (Van Bogaert et al., 2000; De Tiège et al., 2004, 2008). For these analyses, it was necessary to lateralize the epileptogenic zone on the same side in all patients (Van Bogaert et al., 2000). Therefore, we composed a group of left-sided epileptogenic zone patients by selecting either the original scans (16 patients with left focus) or the mirror scans (10 patients with right focus being horizontally flipped) (Van Bogaert et al., 2000). The design matrix included scans of 26 left-lateralized patients (16 original and 10 flipped) and the scans of the 54 control subjects. Separate $t$-contrast analyses identified brain regions where glucose metabolism was significantly lower or higher in the group of patients than in the control population.

Finally, to test the hypothesis of compensatory episodic memory mechanisms in the hemisphere contralateral to the epileptic one, we performed a correlation analysis between regional cerebral glucose metabolism and normalized IAT scores obtained for the injection ipsilateral to the epileptic hemisphere (testing the nonepileptic hemisphere). In practice, in a separate design matrix including the scans of the 
26 left-lateralized patients (16 original scans and 10 flipped scans) taken as one group, normalized "lateralized" IAT scores were introduced as covariates of interest centered around condition means. The normalized lateralized IAT scores consisted of left normalized IAT scores for the 16 patients with left-sided epileptic focus and of right normalized IAT scores for the 10 patients with right-sided epileptic focus whose FDG-PET scans had been flipped. A $t$-contrast analysis was used to search for brain regions showing significant positive correlation between normalized lateralized IAT scores and regional cerebral glucose metabolism in lateralized FDG-PET scans.

In a second step, to avoid the use of flipped scans that assume functional symmetry between brain hemispheres, we performed additional correlation analyses based on a design matrix that included the original scans of 26 patients taken as one group (the 16 scans of patients with left-sided epileptic focus and the 10 nonflipped scans of patients with right-sided epileptic focus). Left and right normalized IAT scores were successively used as covariates of interest centered around condition means. The $t$-contrast analyses identified brain regions showing significant positive correlation between unilateral normalized IAT scores and regional cerebral glucose metabolism. These analyses tested the hypothesis that some brain areas specifically involved in episodic memory neuronal network would show low IAT scores and glucose metabolism level when they were directly involved in the epileptic disorder (ipsilateral to HS) and conversely when not directly involved (contralateral to HS).

Regressions plots between IAT scores and glucose metabolism level at the peak voxels of brain areas showing significant positive correlation in SPM analyses were obtained in Matlab 7.6 R2008a (MathWorks Inc.). After a linear interpolation of the regression plots, the associated slope coefficients and p-values were calculated using Pearson's correlation (corrcoef function, Matlab statistical toolbox version 6.2, Matlab 7.6 R2008a; MathWorks Inc.). Nonparametric correlations were also computed using Spearman's correlation (spear function, Matlab statistical toolbox version 6.2, Matlab 7.6 R2008a; MathWorks Inc.).

All results of SPM analyses were considered significant at $\mathrm{p}<0.05$ corrected for multiple comparisons over the entire brain volume (familywise error, FWE). For individual-level SPM analyses, we also considered significant at a small volume-corrected (SVC) $\mathrm{p}<0.05 \quad(10-\mathrm{mm}$ radius spherical volume of interest) voxels in the mesial and lateral regions of temporal lobes. For correlation analyses, we also considered significant at SVC $\mathrm{p}<0.05$ (10-mm radius spherical volume of interest) brain areas showing positive correlations between normalized IAT scores and glucose metabolism that showed significant increase in metabolism in the nonepileptic temporal lobe at the group level. The anatomic location of significant voxels was determined using the SPM Anatomy Toolbox 1.7b (Institut für
Neurowissenschaften und Medizin [INM], Jülich Forschungszentrum, http://www.fz-juelich.de/inm/inm-1/spm anatomy_toolbox). Pearson's and Spearman's correlation coefficients were considered significant at a value of $\mathrm{p}<0.05$.

\section{ResUlts}

\section{Intracarotid amobarbital test}

In the group of 16 patients with left MTLE, we found a significant difference between right and left IAT scores with a mean score of 6.5 for right IAT scores and of 8.25 for left IAT scores. In the group of 10 patients with right MTLE, we found a significant difference between right and left IAT scores with a mean score of 9.7 for right IAT scores and of 5.7 for left IAT scores.

\section{Individual analyses}

Hypometabolic areas were found in the epileptic temporal lobe in 19 patients (73\%). These areas were located in the amygdalohippocampal complex in 10 patients (FWE: one patient, SVC: nine patients), in the inferior temporal gyrus in eight patients (FWE: six, SVC: two), in the middle superior temporal gyrus in 11 patients (FWE: five, SVC: six), and in the temporal pole in four patients (FWE: two, SVC: two). Areas of significantly increased metabolism were also found in 16 patients $(62 \%)$ in the nonepileptic temporal lobe. These hypermetabolic areas were located in the hippocampus in three patients (SVC), in the inferior temporal gyrus in 12 patients (SVC), in the middle temporal gyrus in four patients (FWE: one patient, SVC: three patients), in the superior temporal gyrus in one patient (SVC), and in the temporal pole in two patients (SVC).

\section{Group analyses}

Significant hypometabolic areas ipsilateral to the epileptogenic zone were found in the amygdalohippocampal complex and parahippocampal gyrus, the inferior and middle temporal gyri, the inferior and middle frontal gyri, and the prefrontal and the cingulate cortex (Fig. 1). In addition, various significant hypermetabolic areas were found contralateral to the epileptic hemisphere and involved the hippocampus, the inferior and superior temporal gyri, the opercular region, and the superior parietal lobule. Bilateral hypermetabolism was also found in the frontal poles (Fig. 2).

\section{Correlation analyses}

The correlation analysis performed between left-lateralized scans and normalized "lateralized" IAT scores showed significant positive correlations between IAT scores and cerebral glucose metabolism in the nonepileptic right hippocampus (entorhinal cortex and Ammon's horn, Fig. 3A,B).

Correlation analyses performed between the original FDG-PET scans and normalized IAT scores showed 


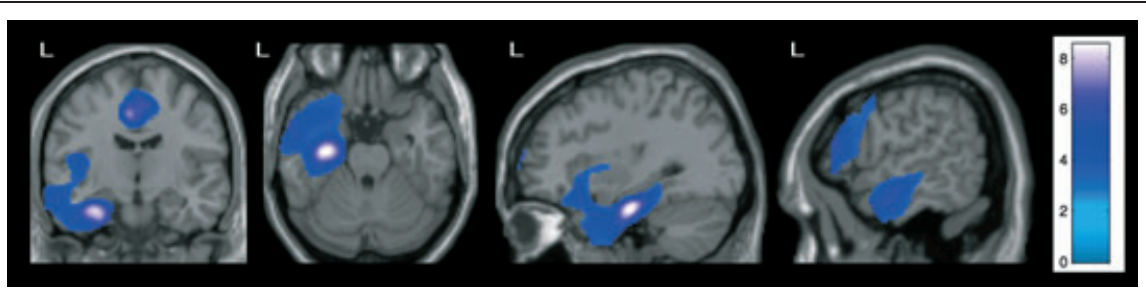

Figure I.

Subtractive SPM analyses at the group level comparing the PET data of the patients taken as a group (left-sided epileptogenic zone) with those of the control group. Significant relative hypometabolic areas ipsilateral to the epileptogenic zone were observed in the amygdalohippocampal complex and parahippocampal gyrus, the inferior and middle temporal gyri, the inferior and middle frontal gyri, and the prefrontal and the cingulate cortex.

Epilepsia (C) ILAE

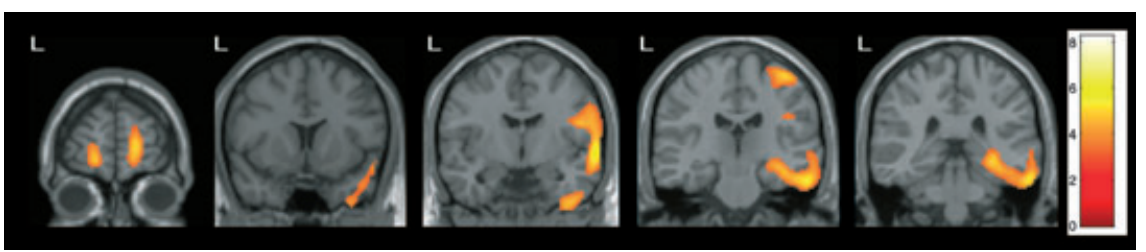

Figure 2.

Subtractive SPM analyses at the group level comparing the PET data of the patients taken as a group (left-sided epileptogenic zone) with those of the control group. Significant relative hypermetabolic areas are observed in the hippocampus, the inferior and superior temporal gyri, the opercular region, and the superior parietal lobule, all contralateral to the epileptic hemisphere and in the frontal pole bilaterally.

Epilepsia C ILAE

significant positive correlations in the mesial and lateral temporal regions contralateral to the injected hemisphere. Normalized left IAT scores showed significant correlation with the cerebral glucose metabolism in the right hippocampus, middle and inferior temporal gyri, and temporal pole (Fig. 4A). The normalized right IAT scores showed significant correlation with the cerebral glucose metabolism in the left hippocampus (Pearson's correlation significant only), superior and inferior temporal gyri, and frontal pole (Fig. 4B). For both right and left IAT scores, regression plots showed that high IAT normalized scores were associated with a high metabolic level in the nonepileptic hippocampus and temporal gyri, whereas low IAT normalized scores were associated with a low metabolic level in the equivalent regions of the epileptic temporal lobe (Fig. 4).

Introducing age, sex, and duration of disease as covariates of no interest in the correlation analyses did not modify the results.

\section{Discussion}

The voxel-based analyses of regional cerebral glucose metabolism performed in a population of 26 patients with MTLE associated with unilateral HS show a significant increase in relative cerebral glucose metabolism in the mesial and lateral structures of the nonepileptic temporal lobe that is positively correlated with the normalized IAT scores.

At the individual level, we found significant relative hypometabolic areas in the epileptic temporal lobe in $73 \%$ of the patients, which is in agreement with previous FDG-PET studies performed in MTLE patients (Engel et al., 1990; Henry et al., 1990, 1993; Theodore et al., 1992; Duncan, 1997; Wieser, 2004). In addition, we found significant increase in relative metabolism in the mesial or lateral temporal regions contralateral to the epileptic focus in $62 \%$ of the patients. Moreover, significant relative hypermetabolic areas were found at the group level in the mesial and lateral temporal regions, the opercular region, and the superior parietal lobule, all in the hemisphere contralateral to the epileptic focus, and in the frontal pole bilaterally. In a previous FDG-PET study using similar SPM methodology but completely different patients and controls populations, we found subthreshold increase in glucose metabolism restricted to the hippocampus contralateral to the epileptic focus (Van Bogaert et al., 2000). In the present study, this contralateral increase in metabolism is more significant and wider than previously found, probably due to the use of larger sets of patients (26 patients vs. 14) and healthy controls (54 controls vs. 27) for the statistical comparison. These two studies highlight the reproducible character of the contralateral increase in metabolism observed in this epileptic disorder. 

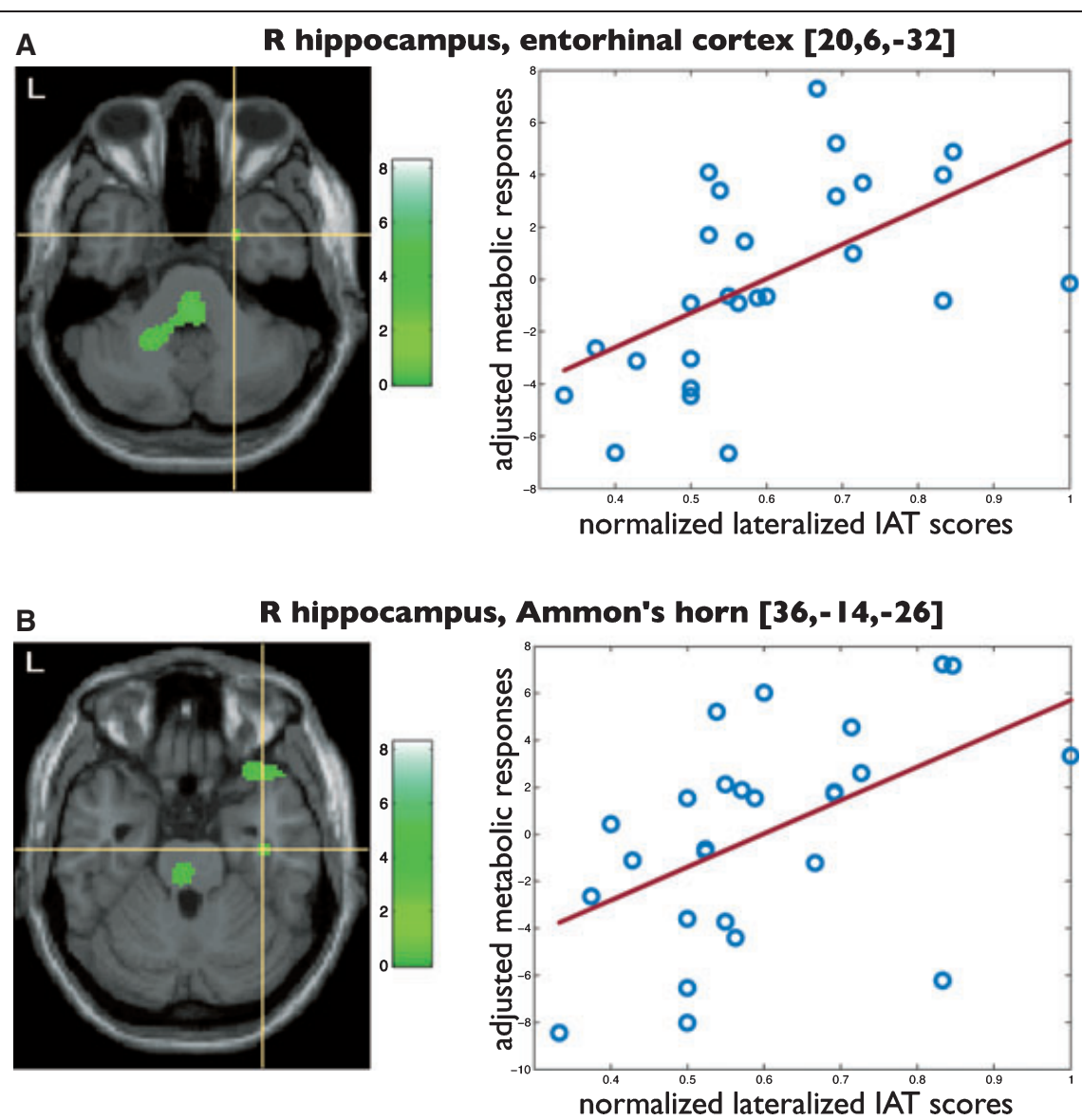

Figure 3.

Results of the correlations analyses performed with scans of left-lateralized patients (left-sided epileptogenic zone) and normalized "lateralized" IAT scores. Positive correlations between normalized lateralized IAT scores and cerebral glucose metabolism were found in the hippocampus of the nonepileptic temporal lobe. Voxels in the right temporal pole and in the infratentorial region were not significant. Regression plots of normalized lateralized IAT scores and adjusted metabolic responses were obtained by considering the peak voxel in $(\mathbf{A})$ the right entorhinal cortex [20, 6, -32] (Pearson's correlation: $r=0.56, p<0.0$ I; Spearman's correlation: $r=0.66, p<10 e^{-4}$ ) and $(\mathbf{B})$ the right Ammon's horn $[36,-14,-26]$ (Pearson's correlation: $r=0.5 I, p<0.01$; Spearman's correlation: $\left.r=0.6 \mathrm{I}, \mathrm{p}<10 \mathrm{e}^{-4}\right)$. These plots showed significant positive correlation between normalized lateralized IAT scores and cerebral glucose metabolism in the considered voxels.

Epilepsia (c) ILAE

Because the mapping analysis of the PET data used in this study is pointing at focal or regional metabolic changes, taking the global brain metabolism as a reference, we might be concerned that this detection of relative hypermetabolic areas actually results from an influence of the hypometabolic changes observed in the hemisphere ipsilateral to the seizure focus on the global brain metabolic estimate (Laurienti, 2004). This type of proportional scaling effect is usually observed in nonaffected brain areas or in brain areas displaying the highest level of metabolic activity at rest such as those belonging to the default-mode network (DMN) (Laurienti, 2004; Yakushev et al., 2009). Such an effect in the present study is unlikely because hypermetabolic areas were limited in size and had specific localizations related to the epileptic condition. In addition, several studies have shown substantial epilepsy-induced functional changes in the nonepileptic temporal lobe in patients with unilateral HS-related MTLE (Bettus et al., 2009, 2010). The correlation found between regional cerebral glucose metabolism and IAT scores also substantiates the results obtained.

After amobarbital injection ipsilateral to the epileptic temporal lobe, we found significantly higher IAT scores than after contralateral injections, in agreement with previous studies performed in similar patient populations (Powell et al., 1987; Rausch et al., 1989; Wyllie et al., 1991). The correlation study between scans of left-lateralized patients and IAT scores showed positive correlations between normalized "lateralized" IAT scores and cerebral glucose 

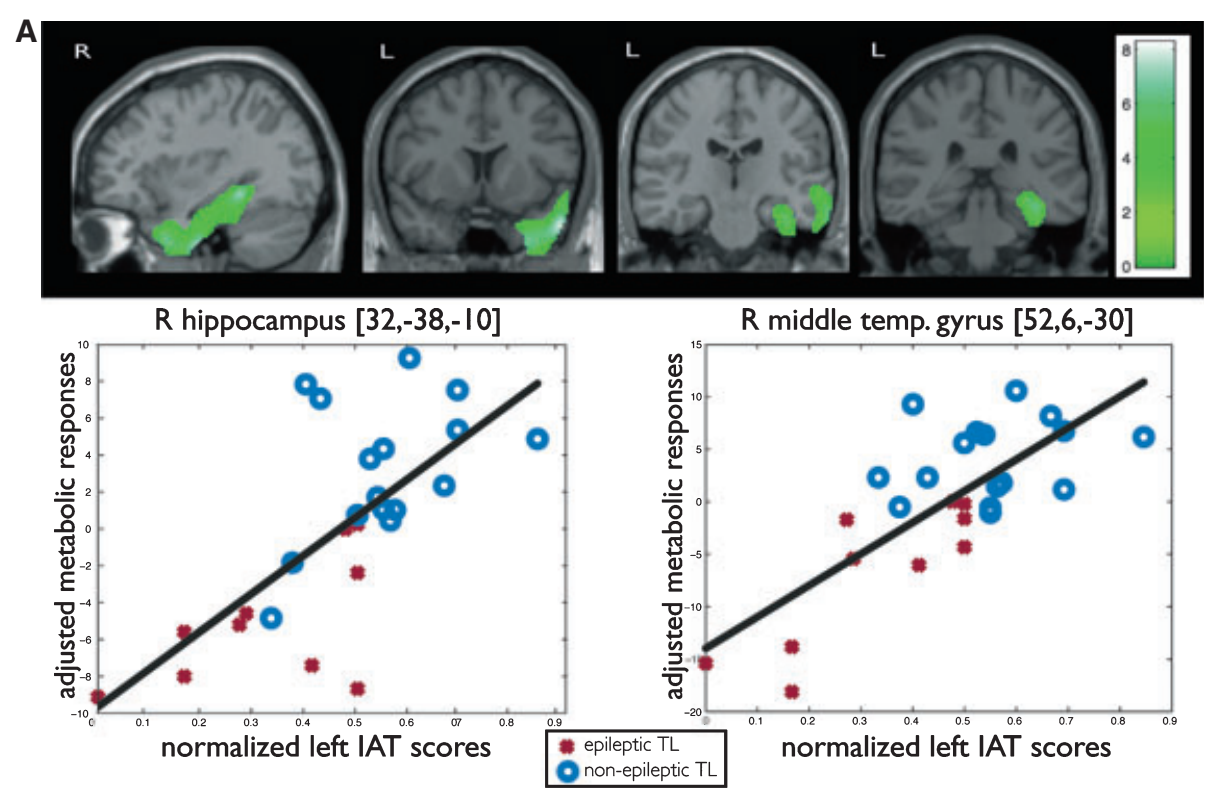

B

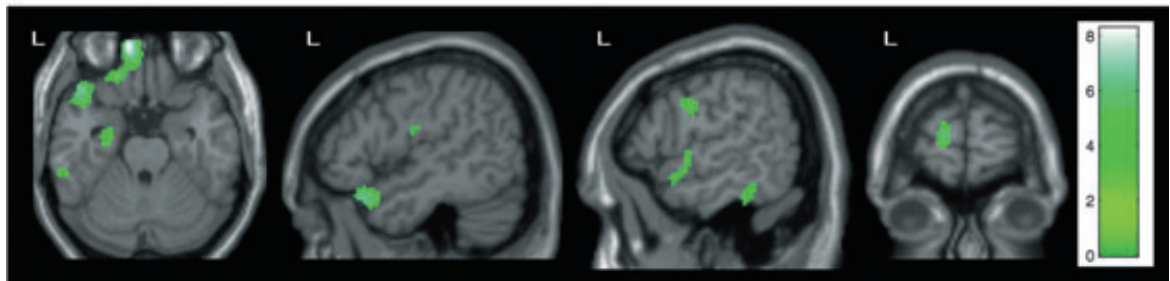

L hippocampus [-30,-12,-22]

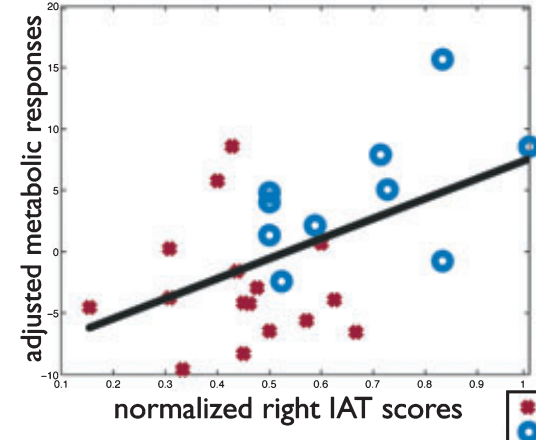

L temporal pole $[-48,26,-26]$

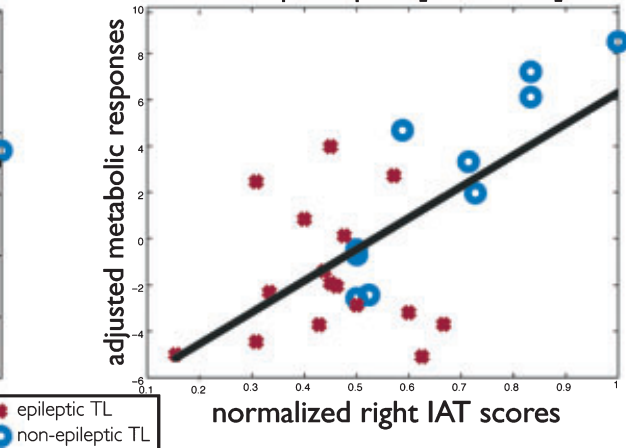

Figure 4.

Results of the correlation analyses performed between the original FDG-PET scans and normalized IAT scores. Positive correlations were found mainly in the mesial and lateral temporal regions contralateral to the injected hemisphere for both left and right IAT scores. Regression plots obtained by considering the peak voxels with significant positive correlation showed that high IAT normalized scores were associated with high metabolic level in the nonepileptic hippocampus and temporal gyri, whereas low IAT normalized scores were associated with low metabolic level in the equivalent regions of the epileptic temporal lobe. (A) Normalized left IAT scores showed significant correlation with the cerebral glucose metabolism in the right hippocampus, middle and inferior temporal gyri, and temporal pole. Regression plots of normalized left IAT scores and adjusted metabolic responses are obtained by considering the peak voxel in the right hippocampus [32, -38, - I0] (Pearson's correlation: $r=0.71$, $p<0.0$ I; Spearman's correlation: $r=0.7 \mathrm{I}, \mathrm{p}<10 \mathrm{e}^{-5}$ ) and in the right middle temporal gyrus [52, 6, -30] (Pearson's correlation: $r=0.76, \mathrm{p}<0.0 \mathrm{I}$; Spearman's correlation: $\left.r=0.63, p<10 e^{-4}\right)$. (B) Normalized right IAT scores showed significant correlation with the cerebral glucose metabolism in the left hippocampus, superior and inferior temporal gyri, and frontal pole. Regression plots of normalized right IAT scores and adjusted responses are obtained by considering the peak voxel in the left hippocampus $[-30,-12,-22]$ (Pearson's correlation: $r=0.49, p<0.05$; Spearman's correlation: $r=0.35, p<0.08$ ) and in the left temporal pole $[-48,26,-26]$ (Pearson's correlation: $r=0.6 \mathrm{I}, \mathrm{p}<0.0 \mathrm{I}$; Spearman's correlation: $r=0.53, \mathrm{p}<0.0 \mathrm{I}$ ).

Epilepsia (C) ILAE 
metabolism in the hippocampus contralateral to the epileptogenic zone. In addition, correlation analyses performed between the original FDG-PET patients' scans and normalized IAT scores showed significant positive correlations in the mesial and lateral temporal regions contralateral to the injected hemisphere for both left and right IAT scores. These results emphasize the key role of mesiotemporal structures in episodic memory functions (Budson \& Price, 2005; Rosenbaum et al., 2005; Leritz et al., 2006; Wang \& Morris, 2010). Moreover, involvement of lateral temporal regions also suggests the functional importance of an access to semantic memory to link conceptual knowledge with the items presented during the IAT procedure. Anterior and lateral temporal cortices indeed represent key structures involved in semantic memory processes (Budson \& Price, 2005; Hodges \& Patterson, 2007). These findings suggest that a relatively high level of metabolism in the nonepileptic temporal lobe might be associated with preserved episodic memory functions after amygdalohippocampectomy. They, therefore, provide a cognitive correlate for the observed temporal hypermetabolism contralateral to the seizure focus, suggesting some degree of epilepsy-induced memory plasticity in the nonepileptic temporal structures of patients with unilateral MTLE. These data are in agreement with preoperative functional MRI (fMRI) studies that investigate memory-encoding processes. These studies indeed showed increased activations in the mesiotemporal regions contralateral to the seizure focus in some MTLE patients, supporting the existence of functional reorganization in the mesiotemporal regions contralateral to the epileptic focus (Jokeit et al., 2001; Richardson et al., 2003; Janszky et al., 2005; Powell et al., 2007, 2008; Cheung et al., 2009). Interestingly, an fMRI study performed in patients with unilateral TLE has shown that the memory performance after unilateral temporal lobe resection was significantly associated with functional activation contralateral to the side of resection, supporting our correlation results obtained in the context of a pharmacologic inactivation of the affected temporal lobe (Cheung et al., 2009). Finally, two interictal resting-state fMRI studies investigating functional connectivity within temporal lobes in patients with MTLE have shown decreased basal functional connectivity within the epileptic temporal lobe contrasting with increased connectivity within the nonepileptic temporal lobe (Bettus et al., 2009, 2010). Interestingly, in one of these studies, the increased basal functional connectivity contralateral to the seizure focus was positively correlated with memory performance, suggesting that the increased wiring within the preserved temporal structures could sustain compensatory memory mechanisms (Bettus et al., 2009).

Two different models of hippocampal functions have been proposed to explain memory deficits after surgery in MTLE patients: the hippocampal reserve and the functional adequacy models (Chelune, 1995). According to the hippocampal reserve model, the capacity of the nonepileptic hip- pocampus to back up determines whether changes in memory function will be observed after surgery. By contrast, the functional adequacy model postulates that it is the part taken by the epileptic hippocampus in memory processes before surgery that determines postoperative memory decline. In the ongoing debate about which model best explains memory deficits after surgery in MTLE patients (Chelune, 1995; Powell et al., 2008; Cheung et al., 2009; Bonelli et al., 2010), our correlation results obtained in the context of a pharmacologic inactivation of the affected temporal lobe brings further support the hippocampal reserve model. Nevertheless, whatever theoretical model is favored, further prospective studies are needed to determine to what extent functional imaging with PET or fMRI might accurately predict, at an individual level, the memory capacity of MTLE patients after surgery.

In conclusion, this study demonstrates the existence of a significant increase in regional cerebral glucose metabolism in the temporal lobe contralateral to the epileptic focus in patients with unilateral MTLE associated with HS. High IAT scores were associated with high metabolic values in the nonepileptic temporal lobe. This finding brings metabolic evidence for the existence of episodic memory plasticity in the nonepileptic temporal lobe of patients with unilateral MTLE.

\section{Acknowledgments}

Noémie Ligot is "clinicien-chercheur doctorant" at the Fonds de la Recherche Scientifique (FRS-FNRS, Belgium). Xavier De Tiège is "clinicien-chercheur spécialiste" at the Fonds de la Recherche Scientifique (FRS-FNRS, Belgium). Koen Van Laere is senior clinical researcher at the FWO (Fonds voor Wetenschappelijk Onderzoek, Belgium). This study was supported by a research grant of the Fonds de la Recherche Scientifique (FRS-FNRS, Belgium).

\section{Disclosure}

The authors have no conflicts of interests. We confirm that we have read the Journal's position on issues involved in ethical publication and affirm that this report is consistent with those guidelines.

\section{REFERENCES}

Akanuma N, Reed LJ, Marsden PK, Jarosz J, Adachi N, Hallett WA, Alarcón G, Morris RG, Koutroumanidis M. (2009) Hemispherespecific episodic memory networks in the human brain: a correlation study between intracarotid amobarbital test and [(18)F]FDG-PET. J Cogn Neurosci 21:605-622.

Berkovic SF, McIntosh AM, Kalnins RM, Jackson GD, Fabinyi GC, Brazenor GA, Bladin PF, Hopper JL. (1995) Preoperative MRI predicts outcome of temporal lobectomy: an actuarial analysis. Neurology 45:1358-1363.

Bettus G, Guedj E, Joyeux F, Confort-Gouny S, Soulier E, Laguitton V, Cozzone PJ, Chauvel P, Ranjeva J-P, Bartolomei F, Guye M. (2009) Decreased basal fMRI functional connectivity in epileptogenic networks and contralateral compensatory mechanisms. Hum Brain Mapp 30:1580-1591.

Bettus G, Bartolomei F, Confort-Gouny S, Guedj E, Chauvel P, Cozzone PJ, Ranjeva JP, Guye M. (2010) Role of resting state functional connec- 
tivity MRI in presurgical investigation of mesial temporal lobe epilepsy. J Neurol Neurosurg Psychiatry 81:1147-1154.

Blume WT, Grabow JD, Darley FL, Aronson AE. (1973) Intracarotid amobarbital test of language and memory before temporal lobectomy for seizure control. Neurology 23:812-819.

Bonelli SB, Powell RH, Yogarajah M, Samson RS, Symms MR, Thompson PJ, Koepp MJ, Duncan JS. (2010) Imaging memory in temporal lobe epilepsy: predicting the effects of temporal lobe resection. Brain 133(Pt 4): 1186-1199.

Brix G, Zaers J, Adam LE, Bellemann ME, Ostertag H, Trojan H, Haberkorn U, Doll J, Oberdorfer F, Lorenz WJ. (1997) Performance evaluation of a whole-body PET scanner using the NEMA protocol. National Electrical Manufacturers Association. J Nucl Med 38:1614-1623.

Budson AE, Price BH. (2005) Memory dysfunction. $N$ Engl J Med 352:692-699.

Chelune GJ. (1995) Hippocampal adequacy versus functional reserve: predicting memory functions following temporal lobectomy. Arch Clin Neuropsychol 10:413-432.

Cheung MC, Chan AS, Lam JM, Chan YL. (2009) Pre- and postoperative fMRI and clinical memory performance in temporal lobe epilepsy. J Neurol Neurosurg Psychiatry 80:1099-1106.

De Tiège X, Goldman S, Laureys S, Verheulpen D, Chiron C, Wetzburger C, Paquier P, Chaigne D, Poznanski N, Jambaque I, Hirsch E, Dulac O, Van Bogaert P. (2004) Regional cerebral glucose metabolism in epilepsies with continuous spikes and waves during sleep. Neurology 63: 853-857.

De Tiège X, Ligot N, Goldman S, Poznanski N, de Saint Martin A, Van Bogaert P. (2008) Metabolic evidence for remote inhibition in epilepsies with continuous spike-waves during sleep. Neuroimage 40:802-810.

Duncan JS. (1997) Imaging and epilepsy. Brain 120(Pt 2):339-377.

Elger CE, Helmstaedter C, Kurthen M. (2004) Chronic epilepsy and cognition. Lancet Neurol 3:663-672.

Engel J Jr, Henry TR, Risinger MW, Mazziotta JC, Sutherling WW, Levesque MF, Phelps ME. (1990) Presurgical evaluation for partial epilepsy: relative contributions of chronic depth-electrode recordings versus FDG-PET and scalp-sphenoidal ictal EEG. Neurology 40:1670 1677.

Engel J Jr, Wiebe S, French J, Sperling M, Williamson P, Spencer D, Gumnit R, Zahn C, Westbrook E, Enos B. (2003) Practice parameter: temporal lobe and localized neocortical resections for epilepsy. Epilepsia 44:741-751.

Gleissner U, Helmstaedter C, Schramm J, Elger CE. (2002) Memory outcome after selective amygdalohippocampectomy: a study in 140 patients with temporal lobe epilepsy. Epilepsia 43:87-95.

Gleissner U, Helmstaedter C, Schramm J, Elger CE. (2004) Memory outcome after selective amygdalohippocampectomy in patients with temporal lobe epilepsy: one-year follow-up. Epilepsia 45:960-962.

Helmstaedter C, Kuthen M, Lux S, Reuber M, Elger CE. (2003) Chronic epilepsy and cognition: a longitudinal study in temporal lobe epilepsy. Ann Neurol 54:425-432.

Henry TR, Mazziotta JC, Engel J Jr, Christenson PD, Zhang JX, Phelps ME, Kuhl DE. (1990) Quantifying interictal metabolic activity in human temporal lobe epilepsy. J Cereb Blood Flow Metab 10:748-757.

Henry TR, Mazziotta JC, Engel J Jr. (1993) Interictal metabolic anatomy of mesial temporal lobe epilepsy. Arch Neurol 50:582-589.

Hodges JR, Patterson K. (2007) Semantic dementia: a unique clinicopathological syndrome. Lancet Neurol 6:1004-1014.

Hong SB, Roh SY, Kim SE, Seo DW. (2000) Correlation of temporal lobe glucose metabolism with the Wada memory test. Epilepsia 41:15541559.

Janszky J, Jokeit H, Kontopoulou K, Mertens M, Ebner A, Pohlmann-Eden B, Woermann FG. (2005) Functional MRI predicts memory performance after right mesiotemporal epilepsy surgery. Epilepsia 46:244-250.

Jokeit H, Ebner A, Arnold S, Schuller M, Antke C, Huang Y, Steinmetz H, Seitz RJ, Witte OW. (1999) Bilateral reductions of hippocampal volume, glucose metabolism, and wada hemispheric memory performance are related to the duration of mesial temporal lobe epilepsy. J Neurol 246:926-933.

Jokeit H, Okujava M, Woermann FG. (2001) Memory fMRI lateralizes temporal lobe epilepsy. Neurology 57:1786-1793.

Laurienti PJ. (2004) Deactivations, global signal, and the default mode of brain function. J Cogn Neurosci 16:1481-1483.
Leritz EC, Grande LJ, Bauer RM. (2006) Temporal lobe epilepsy as a model to understand human memory: the distinction between explicit and implicit memory. Epilepsy Behav 9:1-13.

Loring DW, Lee GP, Meador KJ, Flanigin HF, Smith JR, Figueroa RE, Martin RC. (1990) The intracarotid amobarbital procedure as a predictor of memory failure following unilateral temporal lobectomy. Neurology 40:605-610.

Ojemann GA, Dodrill CB. (1985) Verbal memory deficits after left temporal lobectomy for epilepsy. Mechanism and intraoperative prediction. J Neurosurg 62:101-107.

Perrine K, Gershengorn J, Brown ER, Choi IS, Luciano DJ, Devinsky O. (1993) Material-specific memory in the intracarotid amobarbital procedure. Neurology 43:706-711.

Powell GE, Polkey CE, Canavan AG. (1987) Lateralisation of memory functions in epileptic patients by use of the sodium amytal (Wada) technique. J Neurol Neurosurg Psychiatry 50:665-672.

Powell HW, Richardson MP, Symms MR, Boulby PA, Thompson PJ, Duncan JS, Koepp MJ. (2007) Reorganization of verbal and nonverbal memory in temporal lobe epilepsy due to unilateral hippocampal sclerosis. Epilepsia 48:1512-1525.

Powell HW, Richardson MP, Symms MR, Boulby PA, Thompson PJ, Duncan JS, Koepp MJ. (2008) Preoperative fMRI predicts memory decline following anterior temporal lobe resection. J Neurol Neurosurg Psychiatry 79:686-693.

Rausch R, Babb TL, Engel J, Crandall PH. (1989) Memory following intracarotid amobarbital injection contralateral to hippocampal damage. Arch Neurol 46:783-788.

Richardson MP, Strange BA, Duncan JS, Dolan RJ. (2003) Preserved verbal memory function in left medial temporal pathology involves reorganisation of function to right medial temporal lobe. Neuroimage 20(Suppl. 1):S112-S119.

Rosenbaum RS, Kohler S, Schacter DL, Moscovitch M, Westmacott R, Black SE, Gao F, Tulving E. (2005) The case of K.C.: contributions of a memory-impaired person to memory theory. Neuropsychologia 43:989-1021.

Rosenow F, Luders H. (2001) Presurgical evaluation of epilepsy. Brain 124:1683-1700.

Rouleau I, Robidoux J, Labrecque R, Denault C. (1997) Effect of focus lateralization on memory assessment during the intracarotid amobarbital procedure. Brain Cogn 33:224-241.

Rubin E, Dhawan V, Moeller JR, Takikawa S, Labar DR, Schaul N, Barr WB, Eidelberg D. (1995) Cerebral metabolic topography in unilateral temporal lobe epilepsy. Neurology 45:2212-2223.

Salanova V, Morris HH, Rehm P, Wyllie E, Dinner DS, Lüders H, Gilmore-Pollak W. (1992) Comparison of the intracarotid amobarbital procedure and interictal cerebral 18-fluorodeoxyglucose positron emission tomography scans in refractory temporal lobe epilepsy. Epilepsia 33:635-638

Schuele SU, Luders HO. (2008) Intractable epilepsy: management and therapeutic alternatives. Lancet Neurol 7:514-524.

Spencer DC, Morrell MJ, Risinger MW. (2000) The role of the intracarotid amobarbital procedure in evaluation of patients for epilepsy surgery. Epilepsia 41:320-325.

Theodore WH, Sato S, Kufta C, Balish MB, Bromfield EB, Leiderman DB. (1992) Temporal lobectomy for uncontrolled seizures: the role of positron emission tomography. Ann Neurol 32:789-794.

Van Bogaert P, Massager N, Tugendhaft P, Wikler D, Damhaut P, Levivier M, Brotchi J, Goldman S. (2000) Statistical parametric mapping of regional glucose metabolism in mesial temporal lobe epilepsy. Neuroimage 12:129-138.

Van Paesschen W, Sisodiya S, Connelly A, Duncan JS, Free SL, Raymond AA, Grunewald RA, Revesz T, Shorvon SD, Fish DR, Stevens JM, Johnson CL, Scaravilli F, Harkness WFJ, Jackson GD. (1995) Quantitative hippocampal MRI and intractable temporal lobe epilepsy. Neurology $45: 2233-2240$.

Wada J, Rasmussen T. (1960) Intracarotid injection of sodium amytal for the lateralization of cerebral speech dominance. J Neurosurg 17:266282.

Wang SH, Morris RG. (2010) Hippocampal-neocortical interactions in memory formation, consolidation, and reconsolidation. Annu Rev Psychol 61:49-79, C41-44.

Wiebe S, Blume WT, Girvin JP, Eliasziw M. (2001) A randomized, controlled trial of surgery for temporal-lobe epilepsy. N Engl J Med 345:311-318. 


\section{N. Trotta et al.}

Wieser HG. (2004) ILAE Commission Report. Mesial temporal lobe epilepsy with hippocampal sclerosis. Epilepsia 45:695-714.

Wyllie E, Naugle R, Chelune G, Lüders H, Morris H, Skibinski C. (1991)

Intracarotid amobarbital procedure: II. Lateralizing value in evaluation for temporal lobectomy. Epilepsia 32:865-869.
Yakushev I, Hammers A, Fellgiebel A, Schmidtmann I, Scheurich A, Buchholz HG, Peters J, Bartenstein P, Lieb K, Schreckenberger M. (2009) SPM-based count normalization provides excellent discrimination of mild Alzheimer's disease and amnestic mild cognitive impairment from healthy aging. Neuroimage 44:43-50. 